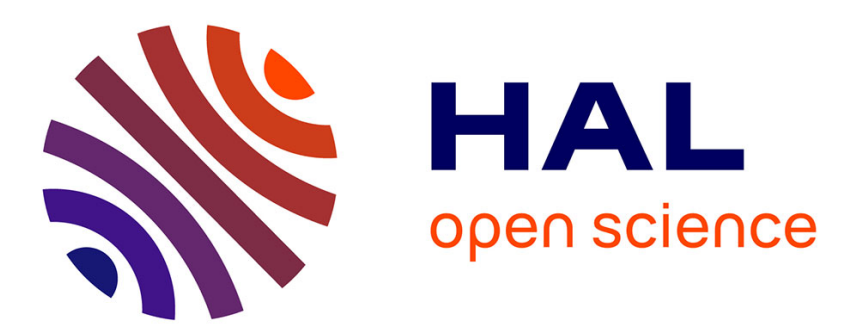

\title{
Transverse dynamics of an intense electron bunch traveling through a pre-ionized plasma
}

R. Lehe, Cédric Thaury, Agustin Lifschitz, Jean-Marcel Rax, Victor Malka

\section{To cite this version:}

R. Lehe, Cédric Thaury, Agustin Lifschitz, Jean-Marcel Rax, Victor Malka. Transverse dynamics of an intense electron bunch traveling through a pre-ionized plasma. Physics of Plasmas, 2014, 21 (4), pp.043104. 10.1063/1.4870336 . hal-01163719

\section{HAL Id: hal-01163719 \\ https://hal.science/hal-01163719}

Submitted on 17 Jul 2015

HAL is a multi-disciplinary open access archive for the deposit and dissemination of scientific research documents, whether they are published or not. The documents may come from teaching and research institutions in France or abroad, or from public or private research centers.
L'archive ouverte pluridisciplinaire HAL, est destinée au dépôt et à la diffusion de documents scientifiques de niveau recherche, publiés ou non, émanant des établissements d'enseignement et de recherche français ou étrangers, des laboratoires publics ou privés. 


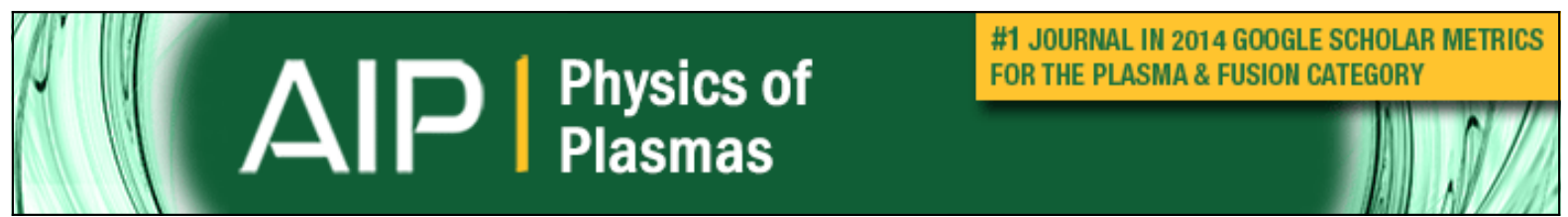

\section{Transverse dynamics of an intense electron bunch traveling through a pre-ionized plasma}

R. Lehe, C. Thaury, A. Lifschitz, J.-M. Rax, and V. Malka

Citation: Physics of Plasmas 21, 043104 (2014); doi: 10.1063/1.4870336

View online: http://dx.doi.org/10.1063/1.4870336

View Table of Contents: http://scitation.aip.org/content/aip/journal/pop/21/4?ver=pdfcov

Published by the AIP Publishing

Articles you may be interested in

Laser plasma formation assisted by ultraviolet pre-ionization

Phys. Plasmas 21, 103511 (2014); 10.1063/1.4898059

Transverse dynamics of a short, relativistic electron bunch in a plasma lens

Phys. Plasmas 2, 2555 (1995); 10.1063/1.871217

Motion of an electron bunch through a plasma

Phys. Fluids 29, 262 (1986); 10.1063/1.865991

Studies of sidescatter and backscatter from pre-ionized plasmas

Phys. Fluids 23, 1319 (1980); 10.1063/1.863144

Large Diameter rf Plasma for Pre-lonization in Confinement Devices

Appl. Phys. Lett. 19, 19 (1971); 10.1063/1.1653726

\section{Did your publisher get}

18 MILLION DOWNLOADS in 2014?

AIP Publishing did.

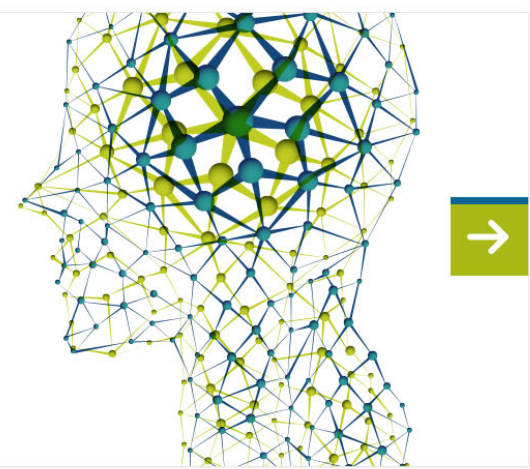




\title{
Transverse dynamics of an intense electron bunch traveling through a pre-ionized plasma
}

\author{
R. Lehe, ${ }^{\text {a) }}$ C. Thaury, A. Lifschitz, J.-M. Rax, and V. Malka \\ Laboratoire d'Optique Appliquée, ENSTA-CNRS-Ecole Polytechnique, UMR 7639, 91761 Palaiseau, France
}

(Received 17 January 2014; accepted 24 March 2014; published online 2 April 2014)

\begin{abstract}
The propagation of a relativistic electron bunch through a plasma is an important problem in both plasma-wakefield acceleration and laser-wakefield acceleration. In those situations, the charge of the accelerated bunch is usually large enough to drive a relativistic wakefield, which then affects the transverse dynamics of the bunch itself. Yet to date, there is no fully relativistic, fully electromagnetic model that describes the generation of this wakefield and its feedback on the bunch. In this article, we derive a model which takes into account all the relevant relativistic and electromagnetic effects involved in the problem. A very good agreement is found between the model and the results of particle-in-cell simulations. The implications of high-charge effects for the transport of the bunch are discussed in detail. (C) 2014 AIP Publishing LLC.

[http://dx.doi.org/10.1063/1.4870336]
\end{abstract}

\section{INTRODUCTION}

Over the past 10 years, the capabilities of laser-wakefield acceleration (LWFA) evolved from hundreds of MeVs (Refs. 1-3) to multi-GeV electron energy. ${ }^{4,5}$ Yet, as these accelerators reach higher and higher energies, there is a growing concern for the preservation of the transverse quality of the bunch (divergence, emittance) throughout the acceleration. Accordingly, a significant effort has recently been put into a theoretical description of the evolution of emittance..$^{6-8}$

One of the standard theoretical representation of laserwakefield acceleration is the fully blown bubble regime. ${ }^{9,10}$ In this model, the transverse focusing fields are the same for all the accelerated electrons. This is true even for a highly charged electron beam, since-in the fully blown regimebeamloading modifies the longitudinal force, ${ }^{11}$ but not the transverse one. ${ }^{12}$ In these conditions, the only source of emittance growth is the finite energy spread (which leads to a progressive betatron decoherence ${ }^{7}$ ).

Yet these results are no longer true when exiting the fully blown bubble regime: if for instance the laser intensity is too weak (linear regime) or the waist too large (quasi-1D non-linear regime), the ponderomotive force is not strong enough to expel all the plasma electrons, and the plasma wake is only partially evacuated. In this case, the intense electron bunch can drive a wakefield of its own, within this partially evacuated cavity. This means in particular that the electrons at the head of the bunch (which drive this wakefield) and the electrons at the tail of the bunch (which are effective already in this wakefield) feel different focusing forces. This effect has strong consequences for the evolution of the transverse size and emittance of the bunch. In plasmawakefield acceleration (PWFA) where the emphasis is mainly on the transverse size of the driving beam, this effect is known to cause head erosion ${ }^{13,14}$ (here with a weak pre-ionizing laser pulse). On the other hand, in LWFA, the

a)remi.lehe@ensta.fr transverse size of the bunch is kept small by the focusing forces of the laser-wakefield anyway, but this beamloading effect can still lead to a growth in emittance. (Notice that we consider the projected emittance here. As mentioned in a related context, ${ }^{15}$ the slice emittance may still be preserved.)

Far from being marginal, this situation in which an electron beam travels behind a pre-ionizing, relatively weak laser pulse is in fact quite common in LWFA:

- In the case of long plasmas, the laser depletes and diffracts before reaching the end of the plasma. Simulations ${ }^{16}$ and experiments ${ }^{17}$ showed that this leads to a transition from a fully blown laser-wakefield to a weak laser-wakefield in which the bunch drives its own bubble. Even for short plasma jets, this is also likely to happen in the end gradient of the jet, where the laser diffracts on a distance $\sim 100 \mu \mathrm{m}$, whereas the density tail usually extends to $\sim 500 \mu \mathrm{m}$. In both cases, it is important to determine to which extend the bunch-driven wakefield will degrade the final transverse properties of the bunch itself.

- In the context of multi-GeV two-stage acceleration, it has been advocated that a quasi-linear laser-wakefield ${ }^{18,19}$ should be used for the second stage-mainly for the sake of stability and controllability. Yet in this case, the emittance of the bunch can be affected by the intrinsic inhomogeneities of the quasi-linear wakefield, ${ }^{6,7}$ but also by the above-mentioned beamloading effects. Regarding the impact of beamloading, the analysis is usually limited to rough estimates (e.g., predicting that they are not too significant for $n_{b} \leq n_{p}$ (Refs. 20 and 21)) and could benefit from a complementary, more rigorous model.

- More generally, recent experimental results ${ }^{22,23}$ suggest that, even for a typical $1 \mathrm{~J}$ self-focused laser pulse, the intensity may not be high enough to blow out all electrons. This implies that, in a significant fraction of past LWFA experiments, the bubble may have been only partially evacuated.

It is thus important to have a formalism to calculate the wakefield that an electron bunch creates in a partially 
evacuated wakefield, and to compute its impact on the transverse dynamics of the bunch itself. Yet to date, no comprehensive satisfying model exists. Rosenzweig et al. ${ }^{24}$ developed an electrostatic and non-relativistic model for the motion of the plasma electrons. This type of model is also used in the Dawson sheet model described in Ref. 9, or in the analysis of head erosion in Ref. 14. However, in the case of laser-wakefield acceleration, the bunch is so intense that it pushes the plasma electrons to relativistic speeds. ${ }^{25}$ In addition to the inertia that the electrons gain by being relativistic, they also become sensitive to the magnetic fields of the bunch, and this has to be accounted for. Moreover, the fast temporal variations of these femtosecond bunches imply that forces that are electromagnetic by nature may appear. On the other hand, Mora and Antonsen ${ }^{26}$ derived a general fully relativistic and electromagnetic framework. This framework has been applied to the case of beamloading in a fully blown bubble, ${ }^{9,12}$ but the case of a partially evacuated wakefield remains to be studied.

In this article, we draw upon the framework of Ref. 26 to derive a model for the evolution of an intense electron bunch in a pre-ionized plasma (e.g., a partially evacuated bubble). This model rigorously takes into account all the relevant relativistic and electromagnetic effects. The article is organized as follows. The general equations of the model are derived from first principles in Sec. II. These equations are then applied to the simple case of a flat-top electron bunch in Sec. III, and their predictions are compared with the results of PIC simulations. Finally, in Sec. IV, these results are used to discuss the transport and acceleration of intense electron bunches in plasmas.

\section{DESCRIPTION OF THE MODEL}

In building this model, we aim to describe the wakefield created by an ultrarelativistic electron bunch propagating behind a pre-ionizing, relatively weak laser pulse. This situation is schematically represented in Fig. 1. More specifically, we are particularly interested in the region immediately surrounding the electron bunch, where the focusing forces that control its transverse dynamics are generated. Three species are considered in the model:

- the immobile plasma ions, which have a uniform and constant density $n_{i}$

- the plasma electrons, which originate from the ionization of the plasma by the laser pulse. They may already have a non-zero longitudinal speed before the bunch reaches them (e.g., at $\xi=0$ on Fig. 1), since they are part of the weakly driven laser-wakefield. Let us denote their density $n_{p}$ and their velocity $\boldsymbol{\beta}_{p}$.

- the ultrarelativistic electron bunch. Let us denote their density $n_{b}$ and their velocity $\boldsymbol{\beta}_{b}$, with $\beta_{b, z} \approx 1$. In typical LWFA applications, this bunch is much denser than the surrounding plasma $\left(n_{b} \gg n_{p}\right)$.

In addition, a few assumptions are made here. First of all, the driving bunch and the resulting wakefield are assumed to be axisymmetric with respect to the axis of propagation. The problem is thus studied in cylindrical

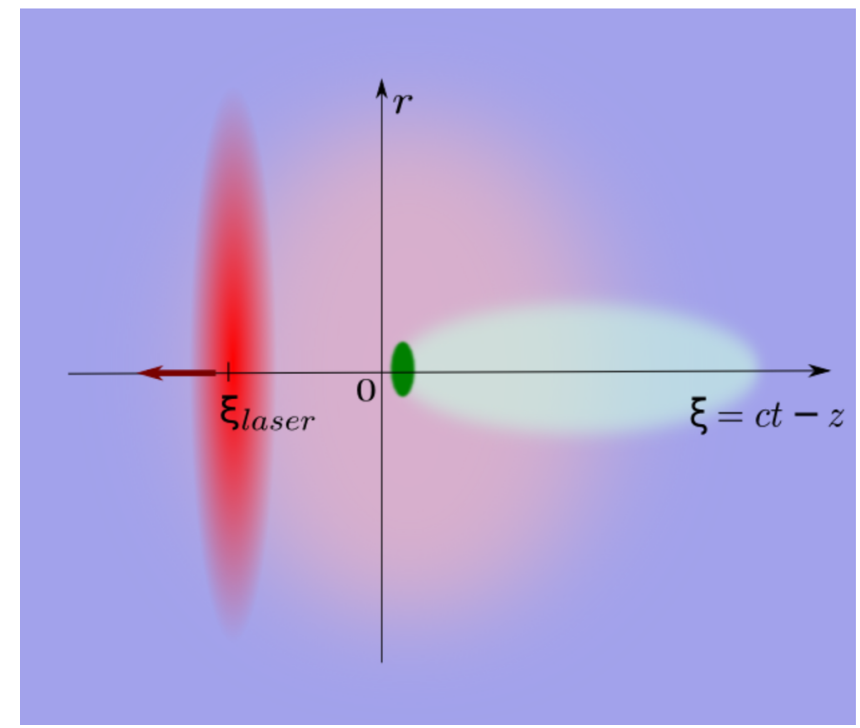

FIG. 1. Schematic representation of the situation considered. Electron density is represented in blue. The laser pulse (red) propagates to the left and drives a weak wakefield (represented as an area of rarefied electron density, and colored in light red). Within this wakefield, the trailing electron bunch (green) drives a stronger wakefield of its own (colored in light green).

coordinate $r, \theta, z$ with $z$ being the coordinate along the axis of propagation.

Since the driving bunch is ultrarelativistic, it is also assumed that it evolves on a timescale much longer than the crossing time of the plasma electrons. This leads to the wellknown quasi-static approximation, in which all wakefield quantities (such as the electric potential $\Phi$, the potential vector $\boldsymbol{A}$, and the trajectories of the plasma electrons) are solely a function of $\xi=c t-z$.

It is also assumed that the trajectories of the plasma electrons do not cross in the neighborhood of the driving bunch. PIC simulations tend to show that these trajectories usually cross a few microns behind the beam. Yet the length of the driving bunch is usually on the order of $1 \mu \mathrm{m}$ or less, and thus trajectory crossing can be neglected when studying the dynamics of the driving bunch.

\section{A. First-principle equations}

Let us consider the normalized potentials $\phi=e \Phi / m c^{2}$ and $\boldsymbol{a}=e \boldsymbol{A} / m c$ in the wakefield. In the Lorenz gauge, the equations for these potentials are

$$
\begin{aligned}
& \left(\nabla^{2}-\frac{1}{c^{2}} \frac{\partial^{2}}{\partial t^{2}}\right) \phi=-4 \pi r_{e}\left[n_{i}-n_{p}-n_{b}\right] \\
& \left(\nabla^{2}-\frac{1}{c^{2}} \frac{\partial^{2}}{\partial t^{2}}\right) \boldsymbol{a}=4 \pi r_{e}\left[n_{p} \boldsymbol{\beta}_{p}+n_{b} \boldsymbol{\beta}_{b}\right],
\end{aligned}
$$

where $r_{e}=e^{2} /\left(4 \pi \epsilon_{0} m c^{2}\right)$ is the classical electron radius. In the quasistatic approximation, $\phi$ and $\boldsymbol{a}$ are functions of $\xi=c t-z$ only, and thus the operators $1 / c^{2} \times \partial_{t}^{2}$ and $\partial_{z}^{2}$ cancel each other. In addition, it is convenient to decompose $\boldsymbol{a}$ into its transverse component $\boldsymbol{a}_{\perp}$ and its longitudinal component $a_{z}$ and to consider the quantity $\psi=\phi-a_{z}$. Thus, $\boldsymbol{a}$ can be expressed as $\boldsymbol{a}=\boldsymbol{a}_{\perp}+(\phi-\psi) \boldsymbol{u}_{z}$ and the field equations become 


$$
\begin{aligned}
\nabla_{\perp}^{2} \phi & =-4 \pi r_{e}\left[n_{i}-n_{p}-n_{b}\right] \\
\nabla_{\perp}^{2} \boldsymbol{a}_{\perp} & =4 \pi r_{e}\left[n_{p} \boldsymbol{\beta}_{p, \perp}+n_{b} \boldsymbol{\beta}_{b, \perp}\right] \\
\nabla_{\perp}^{2} \psi & =-4 \pi r_{e}\left[n_{i}-n_{p}\left(1-\beta_{p, z}\right)-n_{b}\left(1-\beta_{b, z}\right)\right] .
\end{aligned}
$$

Using the cylindrical symmetry of the problem, we obtain

$$
\begin{aligned}
\frac{1}{r} \frac{\partial}{\partial r}\left(r \frac{\partial \phi}{\partial r}\right) & =-4 \pi r_{e}\left[n_{i}-n_{p}-n_{b}\right] \\
\frac{\partial}{\partial r}\left(\frac{1}{r} \frac{\partial\left(r a_{r}\right)}{\partial r}\right) & =4 \pi r_{e}\left[n_{p} \beta_{p, r}+n_{b} \beta_{b, r}\right] \\
\frac{1}{r} \frac{\partial}{\partial r}\left(r \frac{\partial \psi}{\partial r}\right) & =-4 \pi r_{e}\left[n_{i}-n_{p}\left(1-\beta_{p, z}\right)-n_{b}\left(1-\beta_{b, z}\right)\right],
\end{aligned}
$$

where the cylindrical expressions of the Laplacian operator and vector Laplacian operator have been written explicitly.

The potentials $\phi, \psi$, and $a_{r}$ then result in forces that drive the motion of the electrons. In this context, the radial force felt by an electron of the plasma or of the bunch is given by

$$
\begin{aligned}
F_{r} & =-e E_{r}+e c \beta_{z} B_{\theta} \\
& =m c^{2}\left[\frac{1}{c} \frac{\partial a_{r}}{\partial t}+\frac{\partial \phi}{\partial r}+\beta_{z}\left(\frac{\partial a_{r}}{\partial z}-\frac{\partial a_{z}}{\partial r}\right)\right] \\
& =m c^{2}\left[\left(1-\beta_{z}\right)\left(\frac{\partial \phi}{\partial r}+\frac{\partial a_{r}}{\partial \xi}\right)+\beta_{z} \frac{\partial \psi}{\partial r}\right] .
\end{aligned}
$$

\section{B. Approximate system}

The above equations can be simplified by taking into account the specific features of the driving bunch, namely $1-\beta_{b, z} \ll 1$ and $n_{b} \gg n_{p}$. These characteristics imply that the term associated to the driving beam clearly dominates in the equation for $\phi$, but is negligible in the equation for $\psi$. For instance, a bunch containing $100 \mathrm{pC}$ at $200 \mathrm{MeV}$, with a transverse and longitudinal size of $1 \mu \mathrm{m}$ has a density $n_{b}$ $=2 \times 10^{20} \mathrm{~cm}^{-3}$, but $n_{b}\left(1-\beta_{b, z}\right)=2 \times 10^{15} \mathrm{~cm}^{-3}$. By comparison, typical values for $n_{p}$ and $n_{i}$ for tenuous plasmas are of the order $10^{17} \mathrm{~cm}^{-3}-10^{18} \mathrm{~cm}^{-3}$, and PIC simulations show that, in realistic situations, $\left(1-\beta_{p, z}\right)>10^{-1}$. This leads to the simplified field equations

$$
\begin{gathered}
\frac{1}{r} \frac{\partial}{\partial r}\left(r \frac{\partial \phi}{\partial r}\right)=4 \pi r_{e} n_{b}, \\
\frac{\partial}{\partial r}\left(\frac{1}{r} \frac{\partial\left(r a_{r}\right)}{\partial r}\right)=4 \pi r_{e}\left(n_{b} \beta_{b, r}+n_{p} \beta_{p, r}\right), \\
\frac{1}{r} \frac{\partial}{\partial r}\left(r \frac{\partial \psi}{\partial r}\right)=-4 \pi r_{e}\left[n_{i}-n_{p}\left(1-\beta_{p, z}\right)\right] .
\end{gathered}
$$

Notice that we retained both source terms in Eq. (2), since a comparison of $n_{b} \beta_{b, r}$ and $n_{p} \beta_{p, r}$ is inconclusive. This is because for LWFA beams, $\beta_{b, r}$ is of the order $\sim 10^{-2}-10^{-3}$, whereas for the radially expelled plasma electrons, $\beta_{p, r}$ can be of order 1 .

According to the above set of equations, $\phi$ corresponds to the space-charge fields that would be created by the ultrarelativistic bunch if it was in vacuum, while $\psi$ represents the fields generated by the presence of the perturbed plasma (i.e., the wakefield). Because $n_{b} \gg n_{i}$ and $n_{b} \gg n_{p}$, the above system also implies that $\phi \gg \psi$. In this limit, the term $\beta_{z} \partial_{r} \psi$ in the expression of $F_{r}$ is negligible compared to $\left(1-\beta_{z}\right)\left(\partial_{r} \phi+\partial_{\xi} a_{r}\right)$. (A further comparison of the terms $\partial_{r} \phi$ and $\partial_{\xi} a_{r}$ will be carried out in the next section.) The force felt by the plasma electrons thus simplifies to

$$
F_{p, r}=m c^{2}\left(1-\beta_{p, z}\right)\left(\frac{\partial \phi}{\partial r}+\frac{\partial a_{r}}{\partial \xi}\right) .
$$

On the other hand, for the ultrarelativistic electrons of the bunch, the term $\left(1-\beta_{z}\right)$ is vanishingly small and therefore $\left(1-\beta_{z}\right)\left(\partial_{r} \phi+\partial_{\xi} a_{r}\right)$ is negligible compared to $\beta_{z} \partial_{r} \psi$.

$$
F_{b, r}=m c^{2} \frac{\partial \psi}{\partial r} .
$$

A consequence of the above set of approximations is that the plasma electrons only feel the space charge forces of the ultrarelativistic bunch (through $\phi$ ), but do not feel the wakefield forces that are contained in $\psi$. This is clearly not a good approximation when describing the wakefield over a full plasma period, since these wakefield forces are precisely those responsible for the plasma oscillations. Yet, when describing the wakefield over the small length of the driving bunch (which is much shorter than a plasma period), this is a good approximation as these forces do not have enough time to have a substantial impact.

Another consequence of the above approximations is that the electrons of the bunch do not feel the space charge forces of the bunch itself. It is indeed well-known that, due to the compensation of the terms $e \boldsymbol{E}$ and $e \boldsymbol{v} \times \boldsymbol{B}$ in the Lorentz force, these forces are usually negligible for high-energy bunches.

\section{Motion of the plasma electrons}

The radial motion of the plasma electrons is governed by the equation $d_{t}\left(\gamma m v_{p, r}\right)=F_{p, r}$, which can be rewritten as

$$
\frac{d}{d t} \gamma_{p} m \frac{d r_{p}}{d t}=m c^{2}\left(1-\beta_{p, z}\right)\left(\frac{\partial \phi}{\partial r}+\frac{\partial a_{r}}{\partial \xi}\right) .
$$

In the quasistatic approximation, the trajectories of the plasma electrons can be parametrized by $\xi=c t-z$ instead of $t$. Making use of the relation $d_{t}=c\left(1-\beta_{p, z}\right) d_{\xi}$, we obtain

$$
\frac{d}{d \xi} \gamma_{p}\left(1-\beta_{p, z}\right) \frac{d r_{p}}{d \xi}=\left(\frac{\partial \phi}{\partial r}+\frac{\partial a_{r}}{\partial \xi}\right) .
$$

Notice that, in this last operation, the factors $\left(1-\beta_{p, z}\right)$ dropped from both sides of the equation, which physically means that two relativistic effects cancel each other here. The right-hand side factor $\left(1-\beta_{p, z}\right)$ corresponds to the partial compensation of the electric and magnetic forces for a relativistic electron, while the left-hand side $\left(1-\beta_{p, z}\right)$ corresponds to the fact that a relativistic plasma electron tends to travel along with the bunch and thus feels its (diminished) force for a longer time.

In a classical and electrostatic model (e.g., Ref. 24), the dynamics of the electrons is governed by the equation 
$d_{\xi}^{2} r_{p}=\partial_{r} \phi$. Hence, we see that taking into account relativistic dynamics and the full Maxwell equations changes this equation in two ways

- by adding a purely electromagnetic force term $\partial_{\xi} a_{r}$.

- by adding a term $\gamma_{p}\left(1-\beta_{p, z}\right)$ associated with the relativistic dynamics of the electrons.

Let us evaluate the importance of these two terms in typical situations.

A comparison between the terms $\partial_{\xi} a_{r}$ and $\partial_{r} \phi$ can be carried out by first integrating Eqs. (1) and (2). Assuming $\quad a_{r}(r=0)=a_{r}(r=\infty)=0, \partial_{r} a_{r}(r=\infty)=0$ and $\partial_{r} \phi(r=0)=0$ as boundary conditions, this integration leads to

$$
\begin{aligned}
\frac{\partial \phi}{\partial r}= & 4 \pi r_{e}\left(\frac{1}{r} \int_{0}^{r} n_{b}\left(r^{\prime}, \xi\right) r^{\prime} d r^{\prime}\right) \\
\frac{\partial a_{r}}{\partial \xi}= & -\frac{2 \pi r_{e}}{r} \int_{0}^{r} \frac{\partial\left(n_{b} \beta_{b, r}+n_{p} \beta_{p, r}\right)}{\partial \xi}\left(r^{\prime}\right)^{2} d r^{\prime} \\
& -2 \pi r_{e} r \int_{r}^{\infty} \frac{\partial\left(n_{b} \beta_{b, r}+n_{p} \beta_{p, r}\right)}{\partial \xi} d r^{\prime} .
\end{aligned}
$$

If $\beta_{\perp}$ is the typical transverse velocity of the electrons of the bunch, $R$ the typical radial size of the bunch and $L$ its typical length scale along $\xi$, the order of magnitude of the ratio of these terms is

$$
\frac{\partial_{\xi} a_{r}}{\partial_{r} \phi} \sim \frac{R}{L}\left(\beta_{\perp}+\frac{n_{p}}{n_{b}}\right)
$$

where we assume that the radially expelled electrons have $\beta_{p, r} \sim 1$. In typical situations, $\beta_{\perp}<0.01 \mathrm{rad}, n_{p} / n_{b}<10^{-2}$ and the aspect ratio of the bunch $R / L$ is usually less than 10 . The term $\partial_{\xi} a_{r}$ is thus typically negligible.

Let us now evaluate the relative variation of the term $\gamma_{p}\left(1-\beta_{p, z}\right)$. Making use of the conservation of $\gamma_{p}\left(1-\beta_{p, z}\right)$ $-\psi$ in the quasistatic approximation (see Appendix A), one has

$$
\gamma_{p}\left(1-\beta_{p, z}\right)=\gamma_{p, 0}\left(1-\beta_{p, z, 0}\right)+\psi-\psi_{0},
$$

where the index 0 refers to the values of the variables just before reaching the electron bunch (e.g., at $\xi=0$ on Fig. 1). The variation of $\gamma_{p}\left(1-\beta_{p, z}\right)$ is thus equal to $\Delta \psi=\psi-\psi_{0}$. Now, the quantity $\psi$ is maximal inside the electron-driven wakefield, and its highest possible value is reached if the wake is fully evacuated. One may thus find an upper bound for $|\Delta \psi|$ by calculating the value $\Delta \psi_{\max }$ corresponding to a fully blown wakefield of transverse size $R$. By integrating Eq. (3), one finds $\Delta \psi_{\max }=-\pi r_{e} n_{i} R^{2}$. For a driving bunch having a radius $R \sim 1 \mu \mathrm{m}$ and for plasma densities ranging from $n_{i}=10^{18} \mathrm{~cm}^{-3}$ to $n_{i}=10^{17} \mathrm{~cm}^{-3},\left|\Delta \psi_{\max }\right|$ ranges between $10^{-2}$ and $10^{-3}$. On the other hand, for a weakly driven laser-wakefield, $\gamma_{p, 0}\left(1-\beta_{p, z, 0}\right)$ is of order 1 . Hence, we see that the variations of $\gamma_{p}\left(1-\beta_{p, z}\right)$ are usually negligible in the situations that we consider (provided that the density $n_{i}$ is not too big and that the radius $R$ is not too large).

Taking into account all the above considerations, the equation of motion for the plasma electrons reduces to

$$
\frac{d^{2} r_{p}}{d \xi^{2}}=\frac{4 \pi r_{e}}{\gamma_{p, 0}\left(1-\beta_{p, z, 0}\right)}\left(\frac{1}{r_{p}} \int_{0}^{r_{p}} n_{b}\left(r^{\prime}, \xi\right) r^{\prime} d r^{\prime}\right) .
$$

A striking result here is that, although the plasma electrons can be pushed to highly relativistic energies by the space charge of the bunch, their equation of motion-when expressed as a function of $\xi$-is very similar to that of a classical model. ${ }^{24}$ Yet, it differs from it in two important ways:

- It features a constant factor $1 / \gamma_{p, 0}\left(1-\beta_{p, z, 0}\right)$, which takes into account the possible initial relativistic motion of the plasma electrons.

- It neglects the effect of the restoring force of the ions on the motion of the plasma electrons, as discussed in Sec. II B.

Another interesting feature of this equation is that, in order to obtain the trajectories $r_{p}(\xi)$, one does not need to compute the longitudinal dynamics (i.e., the quantities $\beta_{p, z}(\xi), \gamma(\xi)$ ). This greatly simplifies the practical integration of the equation of motion.

\section{Motion of the electrons of the bunch}

Equation (6) implies that the electrons are repelled from the axis by the space charge forces of the beam, thus leaving a depleted zone of low $n_{p}$ near the axis (the wakefield). This generates a field $\psi$ through Eq. (3) which then acts on the electrons of the bunch through the force $-m c^{2} \partial_{r} \psi$ (Eq. (5)). Integrating Eq. (3) leads to

$$
\frac{\partial \psi}{\partial r}=-4 \pi r_{e}\left(\frac{1}{r} \int_{0}^{r}\left[n_{i}-n_{p}\left(r^{\prime}, \xi\right)\left(1-\beta_{p, z}\left(r^{\prime}, \xi\right)\right)\right] r^{\prime} d r^{\prime}\right) .
$$

The evaluation of this force seems to require the calculation of the longitudinal dynamics $\beta_{p, z}$ of the plasma electrons, which we precisely managed to avoid in Sec. II C. This problem can be overcome by noticing that the quantity $\int_{0}^{r_{p}(\xi)} n_{p}\left(1-\beta_{p, z}\right) r^{\prime} d r^{\prime}$ is independent of $\xi$ for any plasma electron trajectory $r_{p}(\xi)$ (see Appendix B). As a result,

$$
\int_{0}^{r} n_{p}\left(1-\beta_{p, z}\right) r^{\prime} d r^{\prime}=\int_{0}^{r_{0}(r, \xi)} n_{p, 0}\left(1-\beta_{p, z, 0}\right) r^{\prime} d r^{\prime},
$$

where $n_{p, 0}$ and $\beta_{p, z, 0}$ are the initial values of $n_{p}$ and $\beta_{p, z}$ and where $r_{0}(r, \xi)$ is the radial position such that a plasma electron which is initially at $r_{0}(r, \xi)$ would reach the radial position $r$ at $\xi$. (Notice that, since the plasma electrons are radially expelled by the bunch, $r_{0}(r, \xi)<r$.) In practice, in order to find $r_{0}(r, \xi)$, one needs to integrate Eq. (6) to find $r_{p}\left(\xi, r_{0}\right)$ and invert the solution. The force applied on the electrons of the bunch can then be calculated, and the equation of motion for these ultrarelativistic electrons becomes

$$
\gamma_{b} m \frac{d^{2} r_{b}}{d t^{2}}=-4 \pi r_{e} m c^{2}\left[\frac{n_{i} r_{b}}{2}-\frac{1}{r_{b}} \int_{0}^{r_{0}\left(r_{b}, \xi\right)} n_{p, 0}\left(1-\beta_{p, z, 0}\right) r^{\prime} d r^{\prime}\right] .
$$

This equation can be compared with the corresponding equations of motion in other models. In a purely electrostatic model, ${ }^{24}$ the term $\int_{0}^{r_{0}(\xi)} n_{p, 0}\left(1-\beta_{p, z, 0}\right) r^{\prime} d r^{\prime}$ is replaced by $\int_{0}^{r_{0}(\xi)} n_{p, 0} r^{\prime} d r^{\prime}$. This is because this type of model neglects the magnetic field produced by the plasma electrons. However, if 
the plasma electrons are relativistic $\left(\left|\beta_{p, z, 0}\right| \approx 1\right)$, they can produce a strong magnetic field, which can significantly modify the total Lorentz force applied on the bunch. On the other hand, in the fully blown bubble regime, ${ }^{9}$ the term $\int_{0}^{r_{0}(\xi)} n_{p, 0}\left(1-\beta_{p, z, 0}\right) r^{\prime} d r^{\prime}$ is neglected altogether, since the bubble is assumed to be completely void of electrons. In this model, the electrons of the bunch feel a force $F_{b, r}$ $=-2 \pi r_{e} m c^{2} n_{i} r=-m \omega_{p}^{2} r / 2$ associated with the bare ion cavity. Yet, in our case, the bunch travels in a partially evacuated cavity and the term $\int_{0}^{r_{0}(\xi)} n_{p, 0}\left(1-\beta_{p, z, 0}\right) r^{\prime} d r^{\prime}$ represents the shielding effect of the plasma electrons over the ions.

In the end, Eqs. (6) and (7), respectively, form the shorttimescale and long-timescale equations of our quasistatic model. Let us give an example of application for this model.

\section{APPLICATION TO A FLAT-TOP BUNCH PROPAGATING IN A LINEAR WAKEFIELD}

In the case of a flat-top bunch propagating in a linear wakefield $\left(a_{0}^{2} \ll 1\right)$, Eq. (6) of our model can be integrated analytically, and a compact expression for the resulting force on the bunch can be obtained. We thus consider a flat-top electron bunch of the form

$$
n_{b}(r, \xi)= \begin{cases}n_{b, 0} & \text { if } 0<\xi<L \text { and } r<R \\ 0 & \text { otherwise }\end{cases}
$$

which propagates behind a weak laser pulse of the form

$\boldsymbol{a}=\left\{\begin{array}{l}a_{0} \cos \left[k_{0}\left(\xi-\xi_{\text {laser }}\right)\right] \cos \left(\frac{\pi}{2} \frac{\left(\xi-\xi_{\text {laser }}\right)}{c \tau}\right) \exp \left(-\frac{r^{2}}{w_{0}^{2}}\right) \boldsymbol{u}_{x} \\ \quad \begin{array}{l}\text { if }\left|\xi-\xi_{\text {laser }}\right|<c \tau \\ 0 \quad \text { otherwise }\end{array}\end{array}\right.$

with $\xi_{\text {laser }}<0$ (as represented in Fig. 1) and $a_{0}^{2} \ll 1$ (linear wakefield). Here, $w_{0}$ is the waist of the laser and $\tau$ is its FWHM duration. We will further assume that $R \ll w_{0}$ (i.e., the size of the bunch is much smaller that the waist of the laser), which is typical in laser-wakefield acceleration.

In this case, the laser-driven wakefield that forms ahead of the bunch $(\xi<0)$ can be calculated analytically. ${ }^{27}$ For our model, the quantities of interest are

$$
\begin{gathered}
\beta_{p, r}=\left(\frac{\eta a_{0}^{2} r}{k_{p} w_{0}^{2}}\right) \cos \left[k_{p}\left(\xi-\xi_{\text {laser }}\right)\right], \\
\beta_{p, z}=-\frac{\eta a_{0}^{2}}{4} \sin \left[k_{p}\left(\xi-\xi_{\text {laser }}\right)\right], \\
n_{p}\left(1-\beta_{p, z}\right)=n_{i}-n_{i} \frac{2 \eta a_{0}^{2}}{k_{p}^{2} w_{0}^{2}} \sin \left[k_{p}\left(\xi-\xi_{\text {laser }}\right)\right],
\end{gathered}
$$

$$
\gamma_{p}=1+O\left(a_{0}^{4}\right),
$$

where $\eta=\pi^{2} \sin \left(\omega_{p} \tau\right) /\left(\pi^{2}-\left(\omega_{p} \tau\right)^{2}\right)$, and where $k_{p}$ is the plasma wavevector associated with the background plasma density: $k_{p}^{2}=4 \pi r_{e} n_{i}$. Note that, in the above equations, we used the assumptions $R \ll w_{0}$ to simplify the expression of the laser wakefield.

\section{A. Motion of the plasma electrons}

In the case of a flat-top bunch, Eq. (6) reduces to

$$
\frac{d^{2} r_{p}}{d \xi^{2}}=\frac{k_{b}^{\prime 2}}{2} r_{p} \quad \text { with } \quad\left\{\begin{array}{l}
k_{b}^{2}=4 \pi r_{e} n_{b, 0} \\
k_{b}^{\prime 2}=\frac{k_{b}^{2}}{1+\eta a_{0}^{2} \sin \left(k_{p}\left|\xi_{\text {laser }}\right|\right) / 4}
\end{array} .\right.
$$

Thus, $k_{b}$ is the Langmuir wavevector of the bunch and $k_{b}^{\prime}$ $=k_{b} / \sqrt{\gamma_{p, 0}\left(1-\beta_{p, z, 0}\right)}$ takes into account the fact that the plasma electrons can be initially mildly relativistic, due to the laser-wakefield. (For instance, for $a_{0}=0.5,\left|\beta_{p, z}\right|$ can reach 0.1 in the laser-wakefield.)

Let us consider a plasma electron which is initially at $r_{0}$ for $\xi=0$. The initial value of the derivative of $r_{p}$ is $\left.d_{\xi} r_{p}\right|_{\xi=0}=\left.d_{t} r_{p}\right|_{\xi=0} /\left[c\left(1-\beta_{p, z, 0}\right)\right]=\beta_{p, r, 0} /\left(1-\beta_{p, z, 0}\right)$. With these initial conditions, the solution reads

$$
\begin{aligned}
r_{p}= & r_{0} \cosh \left(\frac{k_{b}^{\prime} \xi}{\sqrt{2}}\right) \\
& +r_{0}\left(\frac{\sqrt{2} \eta a_{0}^{2} k_{b}^{\prime}}{k_{b}^{2} k_{p} w_{0}^{2}}\right) \cos \left(k_{p} \xi_{\text {laser }}\right) \sinh \left(\frac{k_{b}^{\prime} \xi}{\sqrt{2}}\right)
\end{aligned}
$$

which is valid as long as the electron remains inside the bunch $\left(0<\xi<L, r_{p}<R\right)$. An example of these trajectories is represented in Fig. 2 (The corresponding parameters for the plasma, laser, and accelerated bunch are summed up in Table I).

\section{B. Force acting on the electrons of the bunch}

As explained in Sec. II D, in order to calculate the force applied on the electrons, one should first invert the function $r_{p}\left(r_{0}, \xi\right)$. In the case of Eq. (12), this is straightforward

$$
r_{0}\left(r_{p}, \xi\right)=\frac{r_{p}}{\cosh \left(\frac{k_{b}^{\prime} \xi}{\sqrt{2}}\right)+\left(\frac{\sqrt{2} \eta a_{0}^{2} k_{b}^{\prime}}{k_{b}^{2} k_{p} w_{0}^{2}}\right) \cos \left(k_{p} \xi_{\text {laser }}\right) \sinh \left(\frac{k_{b}^{\prime} \xi}{\sqrt{2}}\right)} .
$$

The force acting on the electrons of the bunch is then derived from Eq. (7), where the term $n_{p, 0}\left(1-\beta_{p, z, 0}\right)$ is obtained from Eq. (10) with $\xi=0$

$$
F_{b, r}=-\frac{m \omega_{p}^{2}}{2} r\left(1-\frac{1+\left(\frac{2 \eta a_{0}^{2}}{k_{p}^{2} w_{0}^{2}}\right) \sin \left(k_{p} \xi_{\text {laser }}\right)}{\left(\cosh \left(\frac{k_{b}^{\prime} \xi}{\sqrt{2}}\right)+\left(\frac{\sqrt{2} \eta a_{0}^{2} k_{b}^{\prime}}{k_{b}^{2} k_{p} w_{0}^{2}}\right) \cos \left(k_{p} \xi_{\text {laser }}\right) \sinh \left(\frac{k_{b}^{\prime} \xi}{\sqrt{2}}\right)\right)^{2}}\right) .
$$



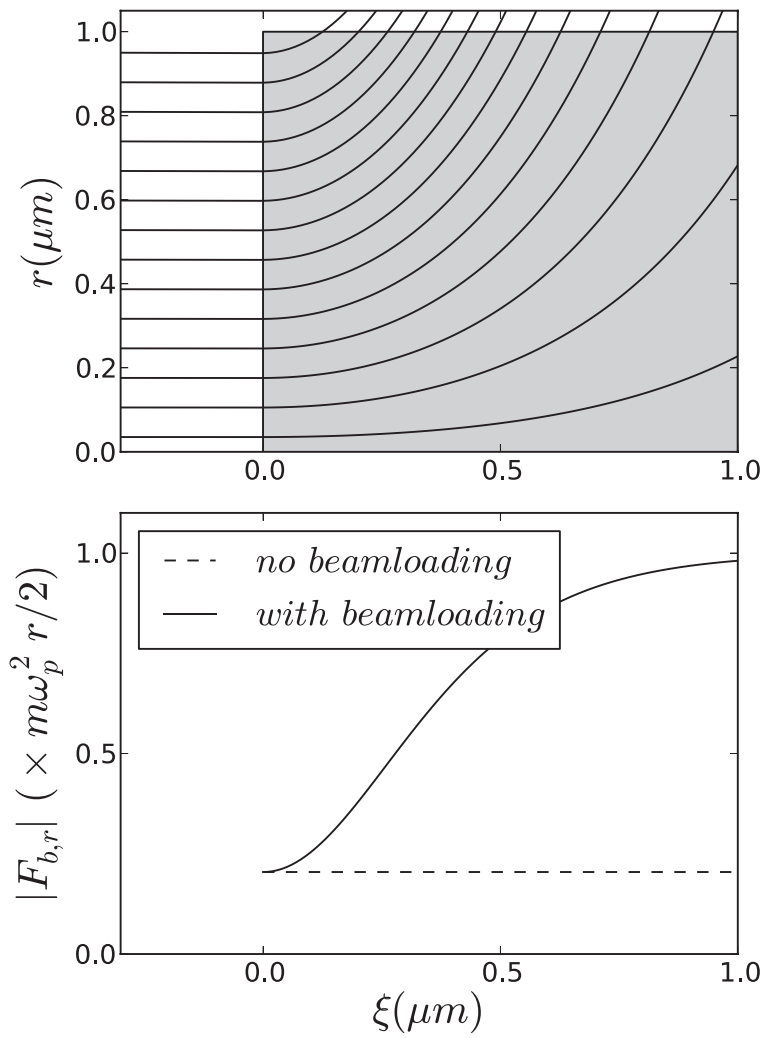

FIG. 2. Top: Trajectories of the plasma electrons (black lines) as given by Eq. (12) and for the parameters of Table I. The flat-top electron bunch is represented by a gray rectangle. Bottom: Radial force acting on the bunch as obtained from Eq. (13), at a given radius $r<R$ (solid line). This force is compared with that of the linear laser-wakefield without beamloading (dashed line). Without beamloading, the force is almost constant over the length of the bunch, since $L \ll \lambda_{p}$.

This force is linear in $r$, but also depends on $\xi$. This expression is plotted in Fig. 2, for the parameters of Table I, with the laser parameters of the left column and the bunch and plasma parameters of the central column (case 1).

\section{Comparison with PIC simulations}

In order to validate the model derived in Sec. II and the corresponding approximations, we compared its predictions for a flat-top bunch (Eqs. (12) and (13)) with the results of PIC simulations.

The simulations were run with the quasi-cylindrical code Calder Circ, ${ }^{28}$ using two azimuthal modes and a spatial resolution $\Delta z=1.3 \times 10^{-2} \mu \mathrm{m}, \Delta r=2.5 \times 10^{-2} \mu \mathrm{m}$. In order to avoid numerical Cherenkov emission by the relativistic electron bunch, we used an extended-stencil Maxwell

TABLE I. Typical laser wakefield parameters, used for Fig. 2 and for the PIC simulations.

\begin{tabular}{lcc}
\hline \hline Laser parameters & Case 1 parameters & Case 2 parameters \\
\hline$w_{0}=10 \mu \mathrm{m}$ & $R=1 \mu \mathrm{m}$ & $R=1 \mu \mathrm{m}$ \\
$\tau=9 \mu \mathrm{m}$ & $L=1 \mu \mathrm{m}$ & $L=1 \mu \mathrm{m}$ \\
$\xi_{\text {laser }}=-10 \mu \mathrm{m}$ & $n_{b, 0}=4 \times 10^{20} \mathrm{~cm}^{-3}$ & $n_{b, 0}=2 \times 10^{20} \mathrm{~cm}^{-3}$ \\
$a_{0}=0.5$ & $n_{i}=1 \times 10^{18} \mathrm{~cm}^{-3}$ & $n_{i}=1 \times 10^{18} \mathrm{~cm}^{-3}$ \\
& $\left(Q_{\text {bunch }}=200 \mathrm{pC}\right)$ & $\left(Q_{\text {bunch }}=100 \mathrm{pC}\right)$ \\
\hline
\end{tabular}

solver similar to that of Cowan et al. ${ }^{29}$ Under the condition $c \Delta t=\Delta z$ (which was indeed verified in our simulations), this solver ensures a perfect dispersion relation along the $z$ axis.

As mentioned in Sec. II, the forces created by the plasma wakefield are very small compared to the space charge fields of the bunch (or the fields of the laser). Therefore, any source of numerical noise in the simulation can easily exceed these forces, and thus prevent a precise comparison with our predictions. For this reason, care has been taken to reduce numerical noise as much as possible. For instance, third-order interpolation factors were used, with 64 macroparticles per cell, initially distributed in a regularly spaced manner.

At the beginning of the simulation, the relativistic flattop bunch is initialized in vaccuum (with $\gamma_{b}=400$ and no radial velocity), and its initial space charge fields are obtained by using the matrix-inversion method of Ref. 29 . The laser is initialized ahead of the bunch, with a moving antenna. Shortly after initialization, the laser and the bunch enter a pre-ionized plasma (a moving window is used).

We start by examining the specific case in which no laser is present $\left(a_{0}=0\right.$; the ultra-relativistic bunch propagates in an unperturbed plasma), and for which our predictions should still be valid. In this case, Eqs. (12) and (13) reduce to

$$
\begin{gathered}
r_{p}=r_{0} \cosh \left(\frac{k_{b} \xi}{\sqrt{2}}\right) \\
F_{b, r}=-\frac{m \omega_{p}^{2}}{2} r \times \tanh \left(\frac{k_{b} \xi}{\sqrt{2}}\right)^{2} .
\end{gathered}
$$

We ran a PIC simulation with no laser $\left(a_{0}=0\right)$ and with the plasma and bunch parameters of the central column of Table I (case 1). Figure 3 shows the trajectories of the plasma electrons as they are repelled by the relativistic bunch and compares them with the predictions of Eq. (14). The simulations and the model are found to be in good agreement. Notice that, as anticipated in the previous

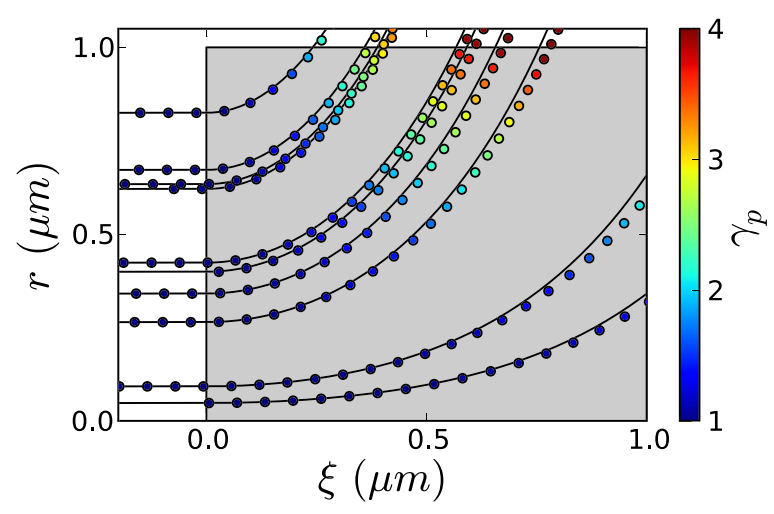

FIG. 3. Trajectories of the plasma electrons in the absence of a laser. The colored dots correspond to the successive positions of a few randomly chosen macroparticles, from the PIC simulations. (The color scale represents the Lorentz factor of the macroparticle.) The black lines correspond to the prediction of Eq. (14), based on the initial radial position $r_{0}$ of each of these macroparticles. 
sections, the plasma electrons do reach relativistic energies $\left(\gamma_{p} \sim 4\right)$ while still lying inside the bunch. This justifies and validates the relativistic approach that has been adopted throughout this article.

The force felt by the electrons of the bunch is plotted in Fig. 4, for the simulation and the model. In the case of the simulation, this force is evaluated by calculating the radial acceleration of the macroparticles: $F_{b r}^{\text {evaluated }} \equiv \gamma_{b} m d_{t}^{2} r_{b}$. There is a pronounced discrepancy between the simulation and the model at the very head $(\xi=0 \mu \mathrm{m})$ and the very tail of the bunch $(\xi=1 \mu \mathrm{m})$. This is most likely due to numerical noise, which was observed to accumulate around the sharp edges of the flat-top bunch. Apart from these localized discrepancies, the model and the simulations are again in good agreement. In particular, as predicted by Eq. (15), the head of the bunch does not experience any focusing in the absence of a laser.

We now turn to the case in which a laser is present. In the corresponding simulation, the parameters of the laser are those of the left column of Table I and the bunch and plasma parameters are those of the right column of Table I (case 2). In particular, a lower bunch charge was chosen in case 2 compared to case 1 , since in this case the evolution of $F_{b, r}$ with $\xi$ is slower and allows a more precise comparison with PIC simulations, despite the presence of numerical noise. The trajectories of the plasma electrons are represented in Fig. 5 and compared with the theoretical predictions of Eq. (12). The simulation data and the model are in excellent agreement. The force felt by the electrons of the bunch is represented in Fig. 6 along with the predictions of Eq. (13) (solid lines). Again, the agreement is good, except at the edges of the bunch-which are presumably more affected by numerical noise. In particular, contrary to the case without a laser (Fig. 4 and Eq. (15)), the head of the bunch does experience a non-zero focusing force, due to the presence of the laser-wakefield. In order to distinctively show this, the data are contrasted with the predictions of Eq. (15) (dashed lines),

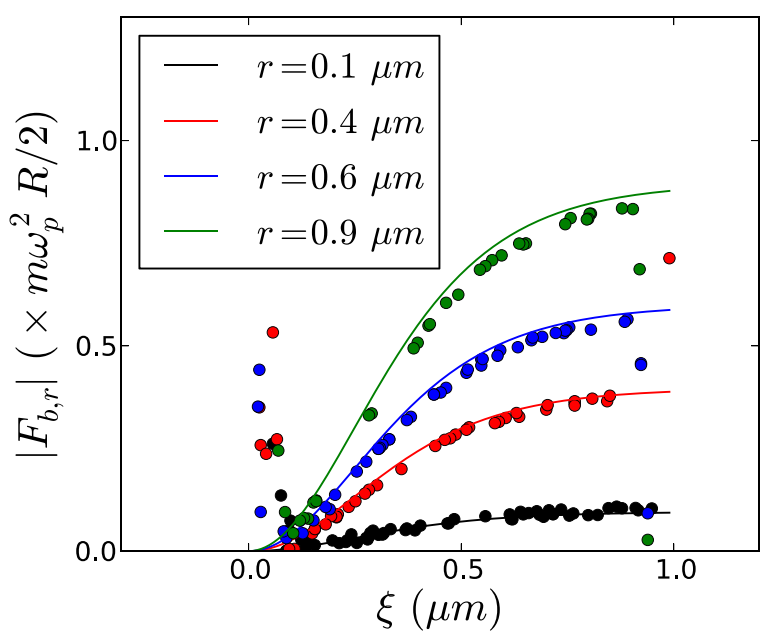

FIG. 4. Force felt by the electrons of the bunch in the absence of a laser, at a given time in the simulation $(\sim 50 \mu \mathrm{m}$ after the entrance in the plasma). The results are plotted for different radii $r$ and each dot corresponds to one macroparticle. (Only the macroparticles lying close to either $r=0.1,0.4$, 0.6 , or $0.9 \mu \mathrm{m}$ have been represented.) The lines correspond to the prediction of Eq. (15).

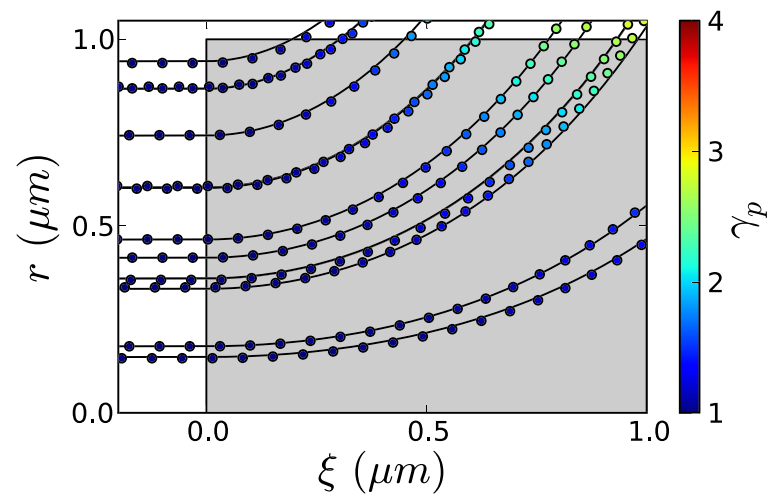

FIG. 5. Trajectories of the plasma electrons in the case where a laser is present. The colored dots correspond to the successive positions of a few randomly chosen macroparticles in the PIC simulation. The black lines correspond to the prediction of Eq. (12), based on the initial radial position $r_{0}$ of each of these macroparticles.

which correspond to the absence of a laser-wakefield and is characterized by a zero focusing force on the head of the bunch.

\section{IMPLICATIONS FOR THE TRANSPORT OF INTENSE BUNCHES IN PLASMAS}

Although Eq. (13) was derived for the specific case of a flat-top bunch in a linear wakefield, some of its qualitative features can be reasonably generalized to other bunch profiles, as well as to the case of a quasi-linear wakefield. One of these features is the qualitative evolution of the focusing force $F_{b, r}$ along the bunch. As shown in Fig. 2, this force transitions between two regimes depending on $\xi$ and $k_{b}^{-1}$. (In Fig. $2, k_{b}^{-1}=0.27 \mu \mathrm{m}$. Notice also that the slight difference between $k_{b}$ and $k_{b}^{\prime}$-which was taken into account in the previous section in order to have precise agreement between simulation and theory-is neglected in the following discussion.)

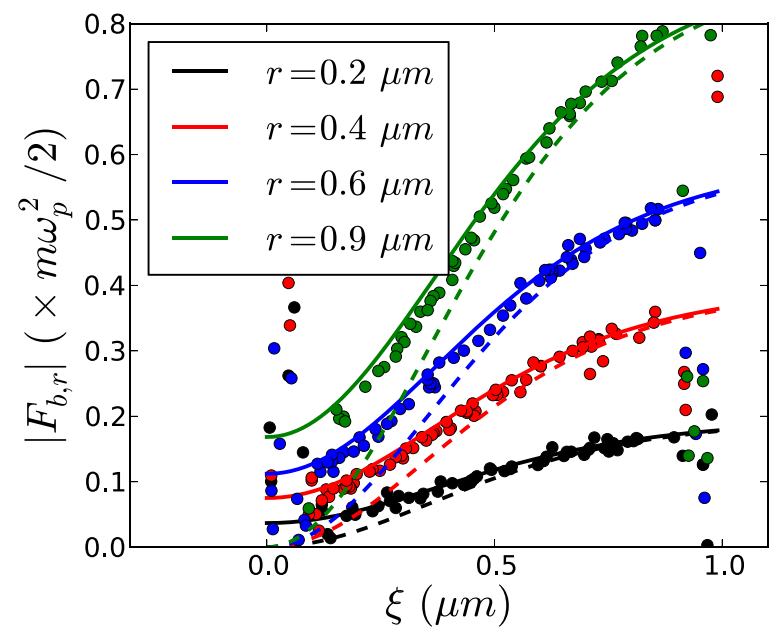

FIG. 6. Force felt by the electrons of the bunch in the presence of a laser, at a given time in the simulation $(\sim 50 \mu \mathrm{m}$ after the entrance in the plasma). The results are plotted for different radii $r$ and each dot corresponds to one macroparticle. The solid lines correspond to the prediction of Eq. (13) with takes into account the presence of the laser-wakefield. For comparison, the dashed lines represent the predictions of Eq. (15), which neglects the presence of the laser-wakefield. 
- For $\xi \ll k_{b}^{-1}$, Eq. (13) reduces to $F_{b, r} \approx-\eta a_{0}^{2}$ $\sin \left(k_{p}\left|\xi_{\text {laser }}\right|\right) \times m c^{2} r / w_{0}^{2}$, which is the usual expression of the focusing force in a linear laser-wakefield and in the absence of beamloading.

- For $\xi \gg k_{b}^{-1}$, Eq. (13) implies that $\left|F_{b, r}\right|$ increases as a function of $\xi$ and eventually saturates at $\left|F_{\max }\right|=m \omega_{p}^{2} r / 2$. This is the standard focusing force inside a fully blown ion cavity, ${ }^{9}$ and it shows that the accelerated beam has repelled all plasma electrons at this point.

Therefore, the overall transverse dynamics of the bunch will be radically different depending on whether the bunch is long enough to experience the second regime. The key parameter here is $k_{b} L$, which can be easily evaluated for a flattop bunch as

$$
k_{b} L=2.6\left(\frac{Q_{\text {bunch }}}{100 \mathrm{pC}}\right)^{1 / 2}\left(\frac{L}{1 \mu \mathrm{m}}\right)^{1 / 2}\left(\frac{R}{1 \mu \mathrm{m}}\right)^{-1} .
$$

In the case of LWFA-generated bunches, typical values are $1 \mathrm{pC}<Q_{\text {bunch }}<200 \mathrm{pC}, 0.3 \mu \mathrm{m}<L<3 \mu \mathrm{m}$ and $0.1 \mu \mathrm{m}$ $<R<3 \mu \mathrm{m}$, and thus $k_{b} L$ can range between 0.05 (short, wide bunch with low charge) and 60 (long, narrow bunch with high charge).

If $k_{b} L>1$, the head of the bunch experiences a purely laser-driven wakefield-which is relatively weak for $a_{0}^{2} \ll 1$-while its tail experiences the strong forces of a purely beam-driven bubble. As shown in Fig. 2, the focusing forces on the head and the tail of the bunch can vary by almost an order of magnitude. This situation is thus very different from that of a low-charge bunch described in Ref. 7. In this reference, the focusing of the head and the tail vary by only a few tens of percent, and this difference is due to the finite energy spread and intrinsic wakefield inhomogeneities (without beamloading). In this case, the degradation of emittance occurs over a distance of $\sim 1 \mathrm{~mm}$, and is nonexistent for an initially matched beam. On the contrary, for $k_{b} L>1$ it is nearly impossible for the beam to be initially matched to the very inhomogeneous focusing forces, and in any realistic case, the emittance will strongly degrade over less than a betatron wavelength.

On the other hand, if $k_{b} L \ll 1$, the situation is more similar to that of Ref. 7, and in fact the beamloading effects described here compete with the effects of finite energy spread and wakefield inhomogeneities. All these effects contribute to betatron decoherence, and each of them leads to a characteristic decoherence distance. In the case of energy spread and wakefield inhomogeneities, their respective expressions are $^{7}$

$$
L_{d \varphi, \Delta \gamma}=\lambda_{\beta} \frac{\gamma_{b}}{\Delta \gamma_{b}} \quad \text { and } \quad L_{d \varphi, \text { inhom. }}=\lambda_{\beta} \frac{\tan \left(k_{p}\left|\xi_{\text {laser }}\right|\right)}{k_{p} L},
$$

where $\lambda_{\beta}=2 \pi w_{0} \sqrt{\gamma_{b}} /\left(\sqrt{\eta \sin \left(k_{p}\left|\xi_{\text {laser }}\right|\right)} a_{0}\right)$ is the betatron wavelength in a linear laser-wakefield. In the case of beamloading, we obtain, based on a Taylor expansion of Eq. (13)

$$
L_{d \varphi, \text { beam. }}=\lambda_{\beta} \frac{4 \eta a_{0}^{2}}{\left(k_{b} L\right)^{2}\left(k_{p} w_{0}\right)^{2}} .
$$

In the context of a linear-wakefield staged accelerator, the above discussion can mitigate previous discussions on the maximal allowable charge density. ${ }^{20,21}$ In these references, it was suggested that the bunch density should be lower than the background plasma density $\left(n_{b}<n_{p}\right)$, so as to avoid blowout and its deleterious consequences on the transverse emittance of the bunch. The above discussion shows that, although blowout does happen for $n_{b}>n_{p}$, its consequences on the emittance of the bunch crucially depend on the length of the bunch. (If the bunch is very short, the inertia of the electrons is such that the blown-out wakefield only develops behind the beam.) In fact, in order to prevent a rapid degradation of transverse emittance, the bunch should satisfy $k_{b} L \ll 1$ (where $k_{b} L$ is given by Eq. (16)) rather than $n_{b}<n_{p}$.

This is particularly important when discussing the effects of beamstrahlung. In Ref. 20, it was shown that reducing the bunch length decreased the deleterious effects of beamstrahlung and was thus desirable. On the other hand, it was also suggested that a smaller bunch length imposed a smaller bunch charge so as to remain below the blowout limit $\left(n_{b}<n_{p}\right)$. On the opposite, here we show that, with our proposed criterion $k_{b} L \ll 1$, decreasing the bunch length does not impose stronger constraints on the bunch charge. (Eq. (16) suggests on the contrary that the constraints on the charge are looser.)

\section{SUMMARY AND CONCLUSION}

In this article, we derived a fully relativistic quasistatic model for the evolution of an electron bunch traveling through a pre-ionized plasma. Throughout the derivation, we made a certain number of approximations, which hold true in the case of LWFA-generated bunches traveling through tenuous plasmas $\left(10^{18}-10^{17} \mathrm{~cm}^{-3}\right)$. This model leads to simple analytical results in the case of a flat-top bunch, and these results were confirmed by PIC simulations.

However, it should be noted that realistic bunches cannot always be approximated by a flat-top distribution, and that, at any rate, even an initially flat-top bunch evolves into other distributions as it travels through a plasma. Yet our model can also be useful in these cases, since it can be numerically integrated, and thereby provides a lightweighted alternative to a full PIC simulation.

Our model can be particularly useful when studying the transverse focusing forces that apply on the bunch. As is wellknown in PWFA, the tail and the head of the bunch do not feel the same focusing forces-a phenomenon which leads to head erosion. Here, we emphasized that a similar phenomenon can occur in LWFA if the laser-wakefield is only partially evacuated. We evaluated the characteristic magnitude and length scale of this effect and showed that it can have a significant degrading impact on the emittance of the bunch. Yet we also showed that this impact crucially depends on the length of the bunch, and that the constraints placed on the charge of the bunch may thus be relaxed for very short bunch.

\section{ACKNOWLEDGMENTS}

We are grateful to S. Corde and X. Davoine for fruitful discussions. This work was partially supported by the European Research Council through the PARIS ERC project (Contract No. 226424). 


\section{APPENDIX A: CONSERVATION OF $\gamma_{p}\left(1-\beta_{p, z}\right)-\psi$}

The conservation of this quantity is a general consequence of the application of the Noether theorem to the quasistatic approximation, and it can also easily be proved in the Hamiltonian formalism (as, e.g., in Ref. 26). An alternative demonstration using the covariant formalism is given here. In covariant notations, the motion of a given plasma electron is given by ${ }^{30}$

$$
m \frac{d u^{\mu}}{d \tau}=-e u_{\nu} F^{\mu \nu}=-e u_{\nu}\left(\partial^{\mu} A^{\nu}-\partial^{\nu} A^{\mu}\right)
$$

with $u^{\mu}=\left(\gamma_{p} c, \gamma_{p} \boldsymbol{v}_{p}\right), A^{\mu}=(\Phi / c, \boldsymbol{A})$ and the convention $\eta_{\mu \nu}=\operatorname{diag}(1,-1,-1,-1)$. As a consequence,

$$
\begin{aligned}
\frac{d \gamma_{p}\left(1-\beta_{p, z}\right)}{d \tau} & =\frac{1}{c} \frac{d\left(u^{0}-u^{3}\right)}{d \tau} \\
& =-\frac{e u_{\nu}}{m c}\left(\partial^{0}-\partial^{3}\right) A^{\nu}+\frac{e u_{\nu}}{m c} \partial^{\nu}\left(A^{0}-A^{3}\right) \\
& =-\frac{e u_{\nu}}{m c}\left(\frac{1}{c} \partial_{t}+\partial_{z}\right) A^{\nu}+\frac{e}{m c} \frac{d}{d \tau}\left(\Phi / c-A_{z}\right) .
\end{aligned}
$$

In the quasistatic appoximation, the fields $\Phi$ and $\boldsymbol{A}$ only depend on $\xi=c t-z$, and thus $\left(1 / c \times \partial_{t}+\partial_{z}\right) A^{\nu}=0$. The above equations thus reduce to

$$
\frac{d}{d \tau}\left[\gamma_{p}\left(1-\beta_{p, z}\right)-\left(\frac{e \Phi}{m c^{2}}-\frac{e A_{z}}{m c}\right)\right]=0 .
$$

Hence, $\gamma_{p}\left(1-\beta_{p, z}\right)-\psi$ is constant.

\section{APPENDIX B: CONSERVATION OF $\int_{0}^{r_{p}(\xi)} n_{p}\left(1-\beta_{p, z}\right) r d r$}

The conservation equation for the plasma electrons reads

$$
\frac{\partial n_{p}}{\partial t}+\nabla \cdot\left(c n_{p} \boldsymbol{\beta}_{p}\right)=0 .
$$

Since $n_{p}$ and $\boldsymbol{\beta}_{p}$ only depend on $\xi=c t-z$ (quasistatic approximation) and $r$ (cylindrical symmetry), this becomes

$$
\frac{\partial}{\partial \xi} n_{p}\left(1-\beta_{p, z}\right)+\frac{1}{r} \frac{\partial}{\partial r}\left(r n_{p} \beta_{p, r}\right)=0 .
$$

Let us use this equation to calculate the variation of $\int_{0}^{r_{p}(\xi)} n_{p}$ $\left(1-\beta_{p, z}\right) r d r$. (Notice that, in this expression, $r_{p}(\xi)$ is a Lagrangian variable describing the trajectory of one given plasma electron, while $n_{p}(r, \xi)$ and $\boldsymbol{\beta}_{p}(r, \xi)$ are Eulerian variables.)

$$
\begin{aligned}
& \frac{d}{d \xi} \int_{0}^{r_{p}(\xi)} n_{p}\left(1-\beta_{p, z}\right) r d r \\
& =\int_{0}^{r_{p}(\xi)} \frac{\partial}{\partial \xi}\left[n_{p}\left(1-\beta_{p, z}\right)\right] r d r+\left(\frac{d r_{p}}{d \xi}\right) n_{p}\left(r_{p}, \xi\right)\left[1-\beta_{p, z}\left(r_{p}, \xi\right)\right] r_{p} \\
& =-\int_{0}^{r_{p}(\xi)} \frac{\partial}{\partial r}\left[r n_{p} \beta_{p, r}\right] d r+\left(\frac{d r_{p}}{d \xi}\right) n_{p}\left(r_{p}, \xi\right)\left[1-\beta_{p, z}\left(r_{p}, \xi\right)\right] r_{p} \\
& =-r_{p} n_{p}\left(r_{p}, \xi\right) \beta_{p, r}\left(r_{p}, \xi\right)+\left(\frac{d r_{p}}{d \xi}\right) n_{p}\left(r_{p}, \xi\right)\left[1-\beta_{p, z}\left(r_{p}, \xi\right)\right] r_{p} .
\end{aligned}
$$

Now, by definition of the Eulerian variable $\boldsymbol{\beta}_{p}$,

$$
\beta_{p, r}\left(r_{p}(\xi), \xi\right) \equiv \frac{1}{c}\left(\frac{d r_{p}}{d t}\right)=\left(1-\beta_{p, z}\right)\left(\frac{d r_{p}}{d \xi}\right)
$$

and thus Eq. (B1) reduces to

$$
\frac{d}{d \xi} \int_{0}^{r_{p}(\xi)} n_{p}\left(1-\beta_{p, z}\right) r d r=0
$$

${ }^{1}$ S. P. D. Mangles, C. D. Murphy, Z. Najmudin, A. G. R. Thomas, J. L. Collier, A. E. Dangor, E. J. Divall, P. S. Foster, J. G. Gallacher, C. J. Hooker, D. A. Jaroszynski, A. J. Langley, W. B. Mori, P. A. Norreys, F. S. Tsung, R. Viskup, B. R. Walton, and K. Krushelnick, Nature 431, 535 (2004).

${ }^{2}$ C. G. R. Geddes, C. Toth, J. van Tilborg, E. Esarey, C. B. Schroeder, D. Bruhwiler, C. Nieter, J. Cary, and W. P. Leemans, Nature 431, 538 (2004).

${ }^{3}$ J. Faure, Y. Glinec, A. Pukhov, S. Kiselev, S. Gordienko, E. Lefebvre, J.-P. Rousseau, F. Burgy, and V. Malka, Nature 431, 541 (2004).

${ }^{4}$ X. Wang, R. Zgadzaj, N. Fazel, Z. Li, S. A. Yi, X. Zhang, W. Henderson, Y.-Y. Chang, R. Korzekwa, H.-E. Tsai, C.-H. Pai, H. Quevedo, G. Dyer, E. Gaul, M. Martinez, A. C. Bernstein, T. Borger, M. Spinks, M. Donovan, V. Khudik, G. Shvets, T. Ditmire, and M. C. Downer, Nat. Commun. 4, Art. No. 1988 (2013).

${ }^{5}$ H. T. Kim, K. H. Pae, H. J. Cha, I. J. Kim, T. J. Yu, J. H. Sung, S. K. Lee, T. M. Jeong, and J. Lee, Phys. Rev. Lett. 111, 165002 (2013).

${ }^{6}$ A. Khachatryan, A. Irman, F. van Goor, and K. Boller, Phys. Rev. ST Accel. Beams 10, 121301 (2007)

${ }^{7}$ T. Mehrling, J. Grebenyuk, F. S. Tsung, K. Floettmann, and J. Osterhoff, Phys. Rev. ST Accel. Beams 15, 111303 (2012).

${ }^{8}$ M. Migliorati, A. Bacci, C. Benedetti, E. Chiadroni, M. Ferrario, A. Mostacci, L. Palumbo, A. R. Rossi, L. Serafini, and P. Antici, Phys. Rev. ST Accel. Beams 16, 011302 (2013).

${ }^{9}$ W. Lu, C. Huang, M. Zhou, M. Tzoufras, F. S. Tsung, W. B. Mori, and T. Katsouleas, Phys. Plasmas 13, 056709 (2006).

${ }^{10}$ A. Pukhov and J. Meyer-ter Vehn, Appl. Phys. B 74, 355 (2002).

${ }^{11}$ C. Rechatin, X. Davoine, A. Lifschitz, A. B. Ismail, J. Lim, E. Lefebvre, J. Faure, and V. Malka, Phys. Rev. Lett. 103, 194804 (2009).

${ }^{12}$ M. Tzoufras, W. Lu, F. S. Tsung, C. Huang, W. B. Mori, T. Katsouleas, J. Vieira, R. A. Fonseca, and L. O. Silva, Phys. Plasmas 16, 056705 (2009).

${ }^{13}$ W. An, M. Zhou, N. Vafaei-Najafabadi, K. A. Marsh, C. E. Clayton, C. Joshi, W. B. Mori, W. Lu, E. Adli, S. Corde, M. Litos, S. Li, S. Gessner, J. Frederico, M. J. Hogan, D. Walz, J. England, J. P. Delahaye, and P. Muggli, Phys. Rev. ST Accel. Beams 16, 101301 (2013).

${ }^{14}$ I. Blumenfeld, "Scaling of the longitudinal electric fields and transformer ratio in a non-linear plasma wakefield accelerator," Ph.D. dissertation (Stanford University, 2009).

${ }^{15}$ C. B. Schroeder, C. Benedetti, E. Esarey, and W. P. Leemans, Phys. Plasmas 20, 123115 (2013).

${ }^{16}$ K. H. Pae, I. W. Choi, and J. Lee, Phys. Plasmas 17, 123104 (2010).

${ }^{17}$ S. Corde, C. Thaury, K. T. Phuoc, A. Lifschitz, G. Lambert, J. Faure, O. Lundh, E. Benveniste, A. Ben-Ismail, L. Arantchuk, A. Marciniak, A. Stordeur, P. Brijesh, A. Rousse, A. Specka, and V. Malka, Phys. Rev. Lett. 107, 215004 (2011).

${ }^{18}$ C. B. Schroeder, E. Esarey, C. G. R. Geddes, C. Benedetti, and W. P. Leemans, Phys. Rev. ST Accel. Beams 13, 101301 (2010).

${ }^{19}$ B. S. Paradkar, B. Cros, P. Mora, and G. Maynard, Phys. Plasmas 20 , 083120 (2013).

${ }^{20}$ C. B. Schroeder, E. Esarey, and W. P. Leemans, Phys. Rev. ST Accel. Beams 15, 051301 (2012).

${ }^{21}$ C. B. Schroeder, E. Esarey, C. Benedetti, and W. P. Leemans, Phys. Plasmas 20, 080701 (2013)

${ }^{22}$ C. Thaury, E. Guillaume, S. Corde, R. Lehe, M. Le Bouteiller, K. Ta Phuoc, X. Davoine, J. M. Rax, A. Rousse, and V. Malka, Phys. Rev. Lett. 111, 135002 (2013). 
${ }^{23}$ S. Corde, C. Thaury, A. Lifschitz, G. Lambert, K. Ta Phuoc, X. Davoine, R. Lehe, D. Douillet, A. Rousse, and V. Malka, Nat. Commun. 4, Art. No. 1501 (2013)

${ }^{24}$ J. B. Rosenzweig, B. Breizman, T. Katsouleas, and J. J. Su, Phys. Rev. A 44, R6189 (1991).

${ }^{25}$ The transverse momentum given by a bunch of charge $Q$ to a plasma electron located at a transverse distance $d$ is of order $m c \frac{Q r_{e}}{e+d}$. For $Q \sim 100 \mathrm{pC}$ and $d \sim 1 \mu \mathrm{m}$ this is of order $m c$.
${ }^{26}$ P. Mora and T. M. Antonsen, Jr., Phys. Plasmas 4, 217 (1997).

${ }^{27}$ E. Esarey, C. B. Schroeder, and W. P. Leemans, Rev. Mod. Phys. 81, 1229 (2009).

${ }^{28}$ A. F. Lifschitz, X. Davoine, E. Lefebvre, J. Faure, C. Rechatin, and V. Malka, J. Comput. Phys. 228, 1803 (2009).

${ }^{29}$ B. M. Cowan, D. L. Bruhwiler, J. R. Cary, E. Cormier-Michel, and C. G. R. Geddes, Phys. Rev. ST Accel. Beams 16, 041303 (2013).

${ }^{30}$ J. D. Jackson, Classical Electrodynamics, 3rd ed. (Wiley, 1998). 